\title{
DYNAMICS OF RATIONAL MAPS: A CURRENT ON THE BIFURCATION LOCUS
}

\author{
Laura DeMarco
}

\begin{abstract}
Let $f_{\lambda}: \mathbf{P}^{1} \rightarrow \mathbf{P}^{1}$ be a family of rational maps of degree $d>1$, parametrized holomorphically by $\lambda$ in a complex manifold $X$. We show that there exists a canonical closed, positive $(1,1)$-current $T$ on $X$ supported exactly on the bifurcation locus $B(f) \subset X$. If $X$ is a Stein manifold, then the stable regime $X-B(f)$ is also Stein. In particular, each stable component in the space

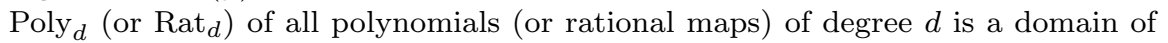
holomorphy.
\end{abstract}

\section{Introduction}

It is well-known that for a rational map $f: \mathbf{P}^{1} \rightarrow \mathbf{P}^{1}$ of degree $d>1$, there is a natural $f$-invariant measure $\mu_{f}$ supported on the Julia set of $f[\mathrm{~B}],[\mathrm{Ly}]$. This measure can be described as the weak limit of purely atomic measures,

$$
\mu_{f}=\lim _{n \rightarrow \infty} \frac{1}{d^{n}} \sum_{\left\{z: f^{n}(z)=a\right\}} \delta_{z},
$$

for any $a \in \mathbf{P}^{1}$ (with at most two exceptions).

There is also a potential-theoretic description of $\mu_{f}$, defined in terms of a homogeneous polynomial lift $F: \mathbf{C}^{2} \rightarrow \mathbf{C}^{2}$ of $f$. The potential function on $\mathbf{C}^{2}$ is given by

$$
h(z)=\lim _{m \rightarrow \infty} \frac{1}{d^{m}} \log \left\|F^{m}(z)\right\|,
$$

and the (1,1)-current $\partial \bar{\partial} h$ satisfies

$$
\pi^{*} \mu_{f}=\frac{i}{\pi} \partial \bar{\partial} h
$$

where $\pi$ is the canonical projection $\mathbf{C}^{2}-\{0\} \rightarrow \mathbf{P}^{1}$ [HP]. In particular, when $f$ is a monic polynomial, this definition reduces to

$$
\mu_{f}=\frac{i}{\pi} \partial \bar{\partial} G=\frac{1}{2 \pi} \Delta G d x \wedge d y
$$

where $G: \mathbf{C} \rightarrow[0, \infty)$ is the Green's function for the complement of the filled Julia set $K(f)=\left\{z: f^{n}(z) \nrightarrow \infty\right.$ as $\left.n \rightarrow \infty\right\}$.

Received March 2, 2000. 
In this paper, we construct a $(1,1)$-current on the parameter space of a holomorphic family of rational maps, supported exactly on the bifurcation locus (just as $\mu_{f}$ is supported exactly on the Julia set).

Let $X$ be a complex manifold. A holomorphic family of rational maps $f$ over $X$ is a holomorphic map $f: X \times \mathbf{P}^{1} \rightarrow \mathbf{P}^{1}$. For each parameter $\lambda \in X$, we obtain a rational map $f_{\lambda}: \mathbf{P}^{1} \rightarrow \mathbf{P}^{1}$ with Julia set $J\left(f_{\lambda}\right)$. The bifurcation locus $B(f)$ of the family $f$ over $X$ is the set of all $\lambda_{0} \in X$ for which $\lambda \mapsto J\left(f_{\lambda}\right)$ is a discontinuous function (in the Hausdorff topology) in any neighborhood of $\lambda_{0}(\S 2)$.

Theorem 1.1. Let $f: X \times \mathbf{P}^{1} \rightarrow \mathbf{P}^{1}$ be a holomorphic family of rational maps on $\mathbf{P}^{1}$ of degree $d>1$. Then there exists a canonical closed, positive (1,1)current $T(f)$ on $X$ such that the support of $T(f)$ is $B(f)$, the bifurcation locus of $f$.

By general properties of positive currents (Lemma 3.3), we have

Corollary 1.2. If $X$ is a Stein manifold, then $X-B(f)$ is also Stein.

Let Rat ${ }_{d}$ and Poly ${ }_{d}$ denote the "universal families" of all rational maps and of all monic polynomials of degree exactly $d>1$. We have Poly ${ }_{d} \simeq \mathbf{C}^{d}$ and Rat $_{d} \simeq \mathbf{P}^{2 d+1}-V$, where $V$ is a resultant hypersurface. In particular, Rat ${ }_{d}$ and $\mathrm{Poly}_{d}$ are Stein manifolds.

Corollary 1.3. Every stable component in Rat $t_{d}$ and Poly is a domain of holomorphy (i.e. a Stein open subset).

Corollary 1.3 answers a question posed by McMullen in [M2], motivated by analogies between rational maps and Teichmüller space. Bers and Ehrenpreis showed that finite-dimensional Teichmüller spaces are domains of holomorphy $[\mathrm{BE}]$.

Sketch proof of Theorem 1.1. Consider a holomorphic family of homogeneous polynomial maps $\left\{F_{\lambda}\right\}$ on $\mathbf{C}^{2}$, locally lifting the holomorphic family $f$ over $X$. Let $\left\{h_{\lambda}\right\}$ be the corresponding potential functions on $\mathbf{C}^{2}$ defined by equation (1). The function $h_{\lambda}(z)$ is plurisubharmonic in both $\lambda \in X$ and $z \in \mathbf{C}^{2}$, and it is pluriharmonic in $z$ away from $\pi^{-1}\left(J\left(f_{\lambda}\right)\right)$. Suppose for simplicity that we have holomorphic functions $c_{j}: X \rightarrow \mathbf{P}^{1}, j=1, \ldots, 2 d-2$, parametrizing the critical points of $f_{\lambda}$ in $\mathbf{P}^{1}$. We choose lifts $\tilde{c}_{j}$ from a neighborhood in $X$ to $\mathbf{C}^{2}$ so that $c_{j}=\pi \circ \tilde{c}_{j}$ and define the plurisubharmonic function

$$
H(\lambda)=\sum_{j} h_{\lambda}\left(\tilde{c}_{j}(\lambda)\right) .
$$

The desired (1,1)-current on $X$ is defined by

$$
T(f)=\frac{i}{\pi} \partial \bar{\partial} H
$$

independent of the choices of $\left\{F_{\lambda}\right\}$ and $\tilde{c}_{j}$. It is supported on $B(f)$ since $H$ fails to be pluriharmonic exactly when a critical point $c_{j}(\lambda)$ passes through the Julia set $J\left(f_{\lambda}\right)$. 
I would like to thank C. McMullen, J.E. Fornaess, and X. Buff for helpful comments and ideas.

\section{Stability}

Let $f: X \times \mathbf{P}^{1} \rightarrow \mathbf{P}^{1}$ be a holomorphic family of rational maps of degree $d>1$. The Julia sets of such a family are said to move holomorphically at a point $\lambda_{0} \in X$ if there is a family of injections $\phi_{\lambda}: J_{\lambda_{0}} \rightarrow \mathbf{P}^{1}$, holomorphic in $\lambda$ near $\lambda_{0}$ with $\phi_{\lambda_{0}}=$ id, such that $\phi_{\lambda}\left(J_{\lambda_{0}}\right)=J_{\lambda}$ and $\phi_{\lambda} \circ f_{\lambda_{0}}(z)=f_{\lambda} \circ \phi_{\lambda}(z)$. In other words, $\phi_{\lambda}$ provides a conjugacy between $f_{\lambda_{0}}$ and $f_{\lambda}$ on their Julia sets. The family of rational maps $f$ over $X$ is stable at $\lambda_{0} \in X$ if any of the following equivalent conditions are satisfied [M1, Theorem 4.2]:

(1) The number of attracting cycles of $f_{\lambda}$ is locally constant at $\lambda_{0}$.

(2) The maximum period of an attracting cycle of $f_{\lambda}$ is locally bounded at $\lambda_{0}$.

(3) The Julia set moves holomorphically at $\lambda_{0}$.

(4) For all $\lambda$ sufficiently close to $\lambda_{0}$, every periodic point of $f_{\lambda}$ is attracting, repelling, or persistently indifferent.

(5) The Julia set $J_{\lambda}$ depends continuously on $\lambda$ (in the Hausdorff topology) in a neighborhood of $\lambda_{0}$.

Suppose also that each of the $2 d-2$ critical points of $f_{\lambda}$ are parametrized by holomorphic functions $c_{j}: X \rightarrow \mathbf{P}^{1}$. Then the following conditions are equivalent to those above:

(6) For each $j$, the family of functions $\left\{\lambda \mapsto f_{\lambda}^{n}\left(c_{j}(\lambda)\right\}_{n \geq 0}\right.$ is normal in some neighborhood of $\lambda_{0}$.

(7) For all nearby $\lambda, c_{j}(\lambda) \in J_{\lambda}$ if and only if $c_{j}\left(\lambda_{0}\right) \in J_{\lambda_{0}}$.

We let $S(f) \subset X$ denote the set of stable parameters and define the bifurcation locus $B(f)$ to be the complement $X-S(f)$. Mañé, Sad, and Sullivan showed that $S(f)$ is open and dense in $X$ [MSS, Theorem A].

Example. In the family $f_{c}(z)=z^{2}+c$, the bifurcation locus is $B(f)=\partial M$, where $M=\left\{c \in \mathbf{C}: f_{c}^{n}(0) \nrightarrow \infty\right.$ as $\left.n \rightarrow \infty\right\}$ is the Mandelbrot set [M1, Theorem 4.6].

Lemma 2.1. If $B(f)$ is contained in a complex hypersurface $D \subset X$, then $B(f)$ is empty.

Proof. Suppose there exists $\lambda_{0} \in B(f)$. By characterization (4) of stability, any neighborhood $U$ of $\lambda_{0}$ must contain a point $\lambda_{1}$ at which the multiplier $m(\lambda)$ of a periodic cycle for $f_{\lambda}$ is passing through the unit circle. In other words, the holomorphic function $m(\lambda)$ defined in a neighborhood $N$ of $\lambda_{1}$ is non-constant with $\left|m\left(\lambda_{1}\right)\right|=1$. The set $\{\lambda \in N:|m(\lambda)|=1\}$ lies in the bifurcation locus and cannot be completely contained in a hypersurface. 


\section{Stein manifolds and positive currents}

Let $X$ be a paracompact complex manifold and $\mathcal{O}(X)$ its ring of holomorphic functions. Then $X$ is a Stein manifold if the following three conditions are satisfied:

- for any $x \in X$ there exists a neighborhood $U$ of $x$ and $f_{1}, \ldots, f_{n} \in \mathcal{O}(X)$ defining local coordinates on $U$;

- for any $x \neq y \in X$, there exists an $f \in \mathcal{O}(X)$ such that $f(x) \neq f(y)$; and

- for any compact set $K$ in $X$, the holomorphic hull

$$
\hat{K}=\left\{x \in X:|f(x)| \leq \sup _{K}|f| \text { for all } f \in \mathcal{O}(X)\right\}
$$

is also compact in $X$.

An open domain $\Omega$ in $X$ is locally Stein if every boundary point $p \in \partial \Omega$ has a neighborhood $U$ such that $U \cap \Omega$ is Stein.

Properties of Stein manifolds. The Stein manifolds are exactly those which can be embedded as closed complex submanifolds of $\mathbf{C}^{N}$. If $\Omega$ is an open domain in $\mathbf{C}^{n}$ then $\Omega$ is Stein if and only if $\Omega$ is pseudoconvex if and only if $\Omega$ is a domain of holomorphy. An open domain in a Stein manifold is Stein if and only if it is locally Stein. Also, an open domain in complex projective space $\mathbf{P}^{n}$ is Stein if and only if it is locally Stein and not all of $\mathbf{P}^{n}$. See, for example, $[\mathrm{H}]$ and the survey article by Siu $[\mathrm{S}]$.

Examples. (1) $\mathbf{C}^{N}$ is Stein. (2) The space of all monic polynomials of degree $d$, Poly $_{d} \simeq \mathbf{C}^{d}$, is Stein. (3) $\mathbf{P}^{n}-V$ for a hypersurface $V$ is Stein. If $V$ is the zero locus of degree $d$ homogeneous polynomial $F$ and $\left\{g_{j}\right\}$ a basis for the vector space of homogeneous polynomials of degree $d$, then the map $\left(g_{1} / F, \ldots, g_{N} / F\right)$ embeds $\mathbf{P}^{n}-V$ as a closed complex submanifold of $\mathbf{C}^{N}$. (4) The space Rat ${ }_{d}$ of all rational maps $f(z)=P(z) / Q(z)$ on $\mathbf{P}^{1}$ of degree exactly $d$ is Stein. Indeed, parameterizing $f$ by the coefficients of $P$ and $Q$ defines an isomorphism Rat $_{d} \simeq \mathbf{P}^{2 d+1}-V$, where $V$ is the resultant hypersurface given by the condition $\operatorname{gcd}(P, Q) \neq 1$.

A $(p, q)$-current $T$ on a complex manifold of dimension $n$ is an element of the dual space to smooth $(n-p, n-q)$-forms with compact support. See [HP], $[\mathrm{Le}]$, and $[\mathrm{GH}]$ for details. The wedge product of a $(p, q)$-current $T$ with any smooth $(n-p, n-q)$-form $\alpha$ defines a distribution by $(T \wedge \alpha)(f)=T(f \alpha)$ for $f \in C_{c}^{\infty}(X)$. Recall that a distribution $\delta$ is positive if $\delta(f) \geq 0$ for functions $f \geq 0$. A $(p, p)$-current is positive if for any system of $n-p$ smooth $(1,0)$-forms with compact support, $\left\{\alpha_{1}, \ldots, \alpha_{n-p}\right\}$, the product

$$
T \wedge\left(i \alpha_{1} \wedge \bar{\alpha}_{1}\right) \wedge \cdots \wedge\left(i \alpha_{n-p} \wedge \bar{\alpha}_{n-p}\right)
$$

is a positive distribution.

An upper-semicontinuous function $h$ on a complex manifold $X$ is plurisubharmonic if $h \mid \mathbf{D}$ is subharmonic for any complex analytic disk $\mathbf{D}^{1}$ in $X$. The current $T=i \partial \bar{\partial} h$ is positive for any plurisubharmonic $h$, and $T \equiv 0$ if and only 
if $h$ is pluriharmonic. The " $\partial \bar{\partial}$-Poincaré Lemma" says that any closed, positive (1,1)-current $T$ on a complex manifold is locally of the form $i \partial \bar{\partial} h$ for some plurisubharmonic function $h[\mathrm{GH}]$.

The next three Lemmas show that the "region of pluriharmonicity" of a plurisubharmonic function is locally Stein. See [C, Theorem 6.2], [U, Lemma 2.4], [FS, Lemma 5.3], and [R, Theorem II.2.3] for similar statements.

Lemma 3.1. Suppose $h$ is plurisubharmonic on the open unit polydisk $\mathbf{D}^{2}$ in $\mathbf{C}^{2}$ and $h$ is pluriharmonic on the "Hartogs domain"

$$
\Omega_{\delta}=\{(z, w):|z|<1,|w|<\delta\} \cup\{(z, w): 1-\delta<|z|<1,|w|<1\} .
$$

Then $h$ is pluriharmonic on $\mathbf{D}^{2}$.

Proof. Let $H$ be a holomorphic function on $\Omega_{\delta}$ such that $h=\operatorname{Re} H$. Any holomorphic function on $\Omega_{\delta}$ extends to $\mathbf{D}^{2}$, and extending $H$ we have $h \leq \operatorname{Re} H$ on $\mathbf{D}^{2}$ since $h$ is plurisubharmonic. The set $A=\left\{z \in \mathbf{D}^{2}: h=\operatorname{Re} H\right\}$ is closed by upper-semi-continuity of $h$. If $A$ has a boundary point $w \in \mathbf{D}^{2}$, then for any ball $B(w)$ about $w$, we have

$$
\begin{aligned}
h(w) & =\operatorname{Re} H(w) \\
& =\frac{1}{|B(w)|} \int_{B(w)} \operatorname{Re} H \\
& >\frac{1}{|B(w)|} \int_{B(w)} h
\end{aligned}
$$

since $\operatorname{Re} H>h$ on a set of positive measure in $B(w)$. This inequality, however, contradicts the sub-mean-value property of the subharmonic function $h$. Therefore $A=\mathbf{D}^{2}$ and $h$ is pluriharmonic on the polydisk.

Lemma 3.2. Let $X$ be a complex manifold. If an open subset $\Omega \subset X$ is not locally Stein, there is $a \delta>0$ and an embedding

$$
e: \mathbf{D}^{2} \rightarrow X
$$

so that $e\left(\Omega_{\delta}\right) \subset \Omega$ but $e\left(\mathbf{D}^{2}\right) \not \subset \Omega$.

Proof. Suppose $\Omega$ is not locally Stein at $x \in \partial \Omega$. By choosing local coordinates in a Stein neighborhood $U$ of $x$ in $X$, we may assume that $U$ is a pseudoconvex domain in $\mathbf{C}^{n}$. Then $\Omega_{0}=U \cap \Omega$ is not pseudoconvex and the function $\phi(z)=$ $-\log d_{0}(z)$ is not plurisubharmonic near $x \in \partial \Omega$. Here, $d_{0}$ is the Euclidean distance function to the boundary of $\Omega_{0}$.

If $\phi$ is not plurisubharmonic at the point $z_{0} \in U \cap \Omega$, then there is a onedimensional disk $\alpha: \mathbf{D}^{1} \rightarrow \Omega$ centered at $z_{0}$ such that $\int_{\partial \mathbf{D}^{1}} \phi<\phi\left(z_{0}\right)$ (identifying the disk with its image $\left.\alpha\left(\mathbf{D}^{1}\right)\right)$. Let $\psi$ be a harmonic function on $\mathbf{D}^{1}$ so that $\psi=\phi$ on $\partial \mathbf{D}^{1}$. Then $\psi\left(z_{0}\right)<\phi\left(z_{0}\right)$. Let $\Psi$ be a holomorphic function on $\mathbf{D}^{1}$ with $\psi=\operatorname{Re} \Psi$.

Now, let $p \in \partial \Omega$ be such that $d_{0}\left(z_{0}\right)=\left|z_{0}-p\right|$. Let $e: \mathbf{D}^{2} \rightarrow U$ be given by

$$
e\left(z_{1}, z_{2}\right)=\alpha\left(z_{1}\right)+z_{2}(1-\varepsilon) e^{-\Psi\left(z_{1}\right)}\left(p-z_{0}\right) .
$$


That is, the two-dimensional polydisk is embedded so that at each point $z_{1} \in \mathbf{D}^{1}$ there is a disk of radius $\left|(1-\varepsilon) \exp \left(-\Psi\left(z_{1}\right)\right)\right|$ in the direction of $p-z_{0}$. If $\varepsilon$ is small enough we have a Hartogs-type subset of the polydisk contained in $\Omega$ but the polydisk is not contained in $\Omega$ since $d_{0}\left(z_{0}, \partial \Omega\right)=\exp \left(-\phi\left(z_{0}\right)\right)<$ $\exp \left(-\psi\left(z_{0}\right)\right)$.

Lemma 3.3. Let $T$ be a closed, positive (1,1)-current on a complex manifold $X$. Then $\Omega=X-\operatorname{supp}(T)$ is locally Stein.

Proof. Let $p$ be a boundary point of $\Omega$. Choose a Stein neighborhood $U$ of $p$ in $X$ so that $T=i \partial \bar{\partial} h$ for some plurisubharmonic function $h$ on $U$. By definition of $\Omega, h$ is pluriharmonic on $U \cap \Omega$.

If $\Omega$ is not locally Stein at $p$, then by Lemma 3.2, we can embed a twodimensional polydisk into $U$ so that a Hartogs-type domain $\Omega_{\delta}$ lies in $\Omega$, but the polydisk is not contained in $\Omega$. By Lemma $3.1, h$ must be pluriharmonic on the whole polydisk, contradicting the definition of $\Omega$.

Corollary 3.4. If $X$ is Stein, then so is $X-\operatorname{supp} T$.

Example. If $X$ is a Stein manifold and $V$ a hypersurface, then $V=\operatorname{supp} T$ for a positive $(1,1)$-current $T$ given locally by $T=\frac{i}{\pi} \partial \bar{\partial} \log |f|$, where $V$ is the zero set of $f$. Lemma 3.3 shows that $X-V$ is locally Stein, and thus Stein. Similarly, $\mathbf{P}^{n}-V$ is Stein for any hypersurface $V$.

\section{The potential function of a rational map}

Let $f: \mathbf{P}^{n} \rightarrow \mathbf{P}^{n}$ be a holomorphic map. Let $F: \mathbf{C}^{n+1} \rightarrow \mathbf{C}^{n+1}$ be a lift of $f$ to a homogeneous polynomial, unique up to scalar multiple, so that $\pi \circ F=f \circ \pi$ where $\pi$ is the projection $\mathbf{C}^{n+1} \backslash 0 \rightarrow \mathbf{P}^{n}$. Let $d$ be the degree of the components of $F$; then $f$ has topological degree $d^{n}$.

Assume that $d>1$. Following [HP], we define the potential function of $F$ by

$$
h_{F}(z)=\lim _{m \rightarrow \infty} \frac{1}{d^{m}} \log \left\|F^{m}(z)\right\| .
$$

The limit converges uniformly on compact subsets of $\mathbf{C}^{n+1}-0$, and $h_{F}(z)$ is plurisubharmonic on $\mathbf{C}^{n+1}$ since $\log \|\cdot\|$ is plurisubharmonic. Let $\Omega_{F} \subset \mathbf{C}^{n+1}$ be the basin of attraction of the origin for $F$; that is,

$$
\Omega_{F}=\left\{x \in \mathbf{C}^{n+1}: F^{m}(x) \rightarrow 0 \text { as } m \rightarrow \infty\right\} .
$$

Note that $\Omega_{F}$ is open and bounded.

From the definition, we obtain the following properties of the potential function $h_{F}[\mathrm{HP}]$ :

(1) $h_{F}(\alpha z)=h_{F}(z)+\log |\alpha|$ for $\alpha \in \mathbf{C}^{*}$;

(2) $\Omega_{F}=\left\{z: h_{F}(z)<0\right\}$; and

(3) $h_{F}$ is independent of the choice of norm $\|\cdot\|$ on $\mathbf{C}^{n+1}$. 
Theorem 4.1. (Hubbard-Papadopol, Ueda, Fornaess-Sibony) The support of the positive $(1,1)$-current

$$
\omega_{f}=\frac{i}{\pi} \partial \bar{\partial} h_{F}
$$

on $\mathbf{C}^{n+1}-0$ is equal to the preimage of the Julia set $\pi^{-1}(J(f))$. If $n=1$, then the Brolin-Lyubich measure $\mu_{f}$ satisfies $\pi^{*} \mu_{f}=\omega_{f}$.

Proof. See [HP, Theorem 4.1] for $n=1$ and [U, Theorem 2.2], [FS, Theorem 2.12] for $n>1$.

From Corollary 3.4, we obtain the following ([U, Theorem 2.3], [FS, Theorem $5.2])$ :

Corollary 4.2. (Ueda, Fornaess-Sibony) The Fatou components of $f: \mathbf{P}^{n} \rightarrow$ $\mathbf{P}^{n}$ are Stein.

\section{The bifurcation current}

In this section we complete the proof of Theorem 1.1. Let $f: X \times \mathbf{P}^{1} \rightarrow \mathbf{P}^{1}$ be a holomorphic family of rational maps on $\mathbf{P}^{1}$ of degree $d>1$. Let $\left\{F_{\lambda}\right\}$ be a holomorphic family of homogeneous polynomials on $\mathbf{C}^{2}$, locally lifting the family $f$, and let $h_{\lambda}$ denote the potential function of $F_{\lambda}(\S 4)$. The potential function $h_{\lambda}(z)$ is plurisubharmonic as a function of the pair $(\lambda, z)$.

Fix $\lambda_{0} \in X$. In a neighborhood $U$ of $\lambda_{0}$, we can choose coordinates on $\mathbf{P}^{1}$ so that $\infty$ is not a critical point of $f_{\lambda}, \lambda \in U$. For $z \in \mathbf{P}^{1}-\{\infty\}$, let $\tilde{z}=(z, 1) \in \mathbf{C}^{2}$. Define a function $H$ on $U$ by

$$
H(\lambda)=\sum_{\left\{c: f_{\lambda}^{\prime}(c)=0\right\}} h_{\lambda}(\tilde{c}),
$$

where the critical points are counted with multiplicity. Now, let $N(\lambda)$ be the number of critical points of the rational map $f_{\lambda}$ (counted without multiplicity). Let

$$
D(f)=\left\{\lambda_{0} \in X: N(\lambda) \text { does not have a local maximum at } \lambda=\lambda_{0}\right\} .
$$

Then $D(f)$ is a complex hypersurface in $X$, since it is defined by the vanishing of a discriminant. If $\lambda_{0} \notin D(f)$, there exists a neighborhood $U$ of $\lambda_{0}$ and holomorphic functions $c_{j}: U \rightarrow \mathbf{P}^{1}, j=1, \ldots, 2 d-2$, parametrizing the critical points of $f_{\lambda}$, such that $\infty \notin c_{j}(U)$ for all $j$. In this case, we can express $H$ as the sum

$$
H(\lambda)=\sum_{j} H_{j}(\lambda)
$$

of the plurisubharmonic functions

$$
\begin{aligned}
H_{j}(\lambda) & =h_{\lambda} \circ \tilde{c}_{j}(\lambda) \\
& =\lim _{m \rightarrow \infty} \frac{1}{d^{m}} \log \left\|F_{\lambda}^{m}\left(\tilde{c}_{j}(\lambda)\right)\right\| .
\end{aligned}
$$

For any $\lambda_{0} \in X$, then, $H$ is defined and continuous in a neighborhood $U$ of $\lambda_{0}$ and plurisubharmonic on $U-D(f)$; therefore $H$ is plurisubharmonic on $U$. 
The bifurcation current $T$ is the positive $(1,1)$-current on parameter space $X$ given locally by

$$
T=\frac{i}{\pi} \partial \bar{\partial} H
$$

The next Lemma shows that $T$ is globally well-defined on $X$.

Lemma 5.1. The current $T=\frac{i}{\pi} \partial \bar{\partial} H$ is independent of (a) the choice of lifts $\tilde{c}_{j}$ of $c_{j}$ and (b) the choice of lifts $F_{\lambda}$ of $f_{\lambda}$.

Proof. Suppose we define a new lift $\hat{c}_{j}(\lambda)=t(\lambda) \cdot \tilde{c}(\lambda)$ for some holomorphic function $t$ taking values in $\mathbf{C}^{*}$. Property (1) of the potential function $h_{\lambda}(\S 4)$ implies that $h_{\lambda}(\hat{c}(\lambda))=h_{\lambda}(\tilde{c}(\lambda))+\log |t(\lambda)|$ and $i \partial \bar{\partial} H$ is unchanged since $\log |t(\lambda)|$ is pluriharmonic, proving (a). If the lifted family $\left\{F_{\lambda}\right\}$ is similarly replaced by $\left\{t(\lambda) \cdot F_{\lambda}\right\}$, a computation shows that $h_{\lambda}$ is changed only by the addition of the pluriharmonic term $\frac{1}{d-1} \log |t(\lambda)|$ where $d$ is the degree of the $f_{\lambda}$. This proves (b).

Lemma 5.2. A parameter $\lambda_{0}$ lies in the stable regime $S(f) \subset X$ if and only if the function $H$ is pluriharmonic in a neighborhood of $\lambda_{0}$.

Proof. Let us first suppose that $\lambda_{0} \in S(f)$ is not in $D(f)$ (in the notation above). By characterization (6) of stability ( $(2)$, for each $j$, the family of functions $\left\{\lambda \mapsto f_{\lambda}^{m}\left(c_{j}(\lambda)\right)\right\}$ is normal in a neighborhood $V$ of $\lambda_{0}$; hence, there exists a subsequence converging uniformly on compact subsets to a holomorphic function $g_{j}(\lambda)$. As in [HP, Prop 5.4], we can shrink our neighborhood $V$ if necessary to find a norm $\|\cdot\|$ on $\mathbf{C}^{2}$ so that $\log \|\cdot\|$ is pluriharmonic on $\pi^{-1}\left(g_{j}(V)\right)$; e.g., if $g_{j}(V)$ is disjoint from $\{|x|=|y|\}$, we can choose norm $\|(x, y)\|=\max \{|x|,|y|\}$. Then, on any compact set in $V$, the functions

$$
\lambda \mapsto \frac{1}{d^{m_{k}}} \log \left\|F_{\lambda}^{m_{k}}\left(\tilde{c}_{j}(\lambda)\right)\right\|
$$

are pluriharmonic if $k$ is large enough. By property (3) of the potential function $h_{\lambda}(\S 4)$, this subsequence converges uniformly to $H_{j}$. Therefore, $H$ is pluriharmonic on $V$.

If $\lambda_{0}$ lies in $D(f) \cap S(f)$, then $H$ is defined and continuous on a neighborhood $V$ of $\lambda_{0}$ and pluriharmonic on $V-D(f)$. As $D(f)$ has codimension $1, H$ must be pluriharmonic on all of $V$.

For the converse, let us suppose again that $\lambda_{0} \notin D(f)$ and that $H$ is pluriharmonic in a neighborhood of $\lambda_{0}$. Each $H_{j}$ is pluriharmonic and so we may write $H_{j}=\operatorname{Re} G_{j}$ in a neighborhood $V$ of $\lambda_{0}$. In analogy with [U, Prop. 2.1], we define new lifts $\hat{c}_{j}(\lambda)=e^{-G_{j}(\lambda)} \cdot \tilde{c}(\lambda)$ of the $c_{j}$ and compute

$$
\begin{aligned}
h_{\lambda}\left(\hat{c}_{j}(\lambda)\right) & =h_{\lambda}(\tilde{c}(\lambda))+\log \left|e^{-G_{j}(\lambda)}\right| \\
& =h_{\lambda}(\tilde{c}(\lambda))-\operatorname{Re} G_{j} \\
& =H_{j}-H_{j} \\
& =0 .
\end{aligned}
$$


By property (2) of $h_{\lambda}$, this implies that $\hat{c}_{j}(\lambda)$ lies in $\partial \Omega_{\lambda}$ for all $\lambda \in V$. If $V$ is small enough, the set $\cup_{\lambda \in V}\left(\{\lambda\} \times \partial \Omega_{\lambda}\right)$ has compact closure in $X \times \mathbf{C}^{2}$. As the functions $F_{\lambda}$ preserve $\partial \Omega_{\lambda}$, the family $\left\{\lambda \mapsto F_{\lambda}^{n}\left(\hat{c}_{j}(\lambda)\right)\right\}$ is uniformly bounded and thus normal. Of course, $f_{\lambda}^{n} \circ c_{j}=\pi \circ F_{\lambda}^{n} \circ \hat{c}_{j}$ demonstrating that $\lambda_{0}$ is a stable parameter by (6) of Section 2 .

Finally suppose that $H$ is pluriharmonic in a neighborhood $U$ of parameter $\lambda_{0} \in D(f)$. Then $U-D(f)$ lies in the stable regime and Lemma 2.1 shows that all of $U$ must belong to $S(f)$.

Proof of Theorem 1.1. Let $T$ be the bifurcation current defined above for the family of rational maps $f$ over $X$. By Lemma 5.2, the support of $T$ is the bifurcation locus $B(f)$.

Corollaries 1.2 and 1.3 now follow immediately from Corollary 3.4.

\section{Examples}

Example 6.1. In the family $\left\{f_{\lambda}(z)=z^{d}+\lambda\right\}, \lambda \in \mathbf{C}$, the bifurcation current $T$ takes the form

$$
T=\frac{d-1}{d}\left(\frac{i}{\pi} \partial \bar{\partial} G\right)
$$

where $G$ is the Green's function for the complement of the "degree $d$ Mandelbrot set" $M_{d}=\left\{\lambda: f_{\lambda}^{n}(0) \nrightarrow \infty\right.$ as $\left.n \rightarrow \infty\right\}$. That is, $T$ is a multiple of harmonic measure supported on $\partial M_{d}$. The $T$-mass of $\partial M_{d}$ is $(d-1) / d$.

Proof. If $G_{\lambda}$ denotes the Green's function for the complement of the filled Julia set $K\left(f_{\lambda}\right)=\left\{z: f_{\lambda}^{n}(z) \nrightarrow \infty\right.$ as $\left.n \rightarrow \infty\right\}$, then $G(\lambda)=G_{\lambda}(\lambda)$ (see e.g. [CG, VIII.4]). By [HP, Prop 8.1], we have

$$
h_{\lambda}(x, y)=G_{\lambda}(x / y)+\log |y|
$$

where $(x, y), y \neq 0$, is a point of $\mathbf{C}^{2}$. Note that $d-1$ of the critical points of $f_{\lambda}$ are at $z=0$ and the other $d-1$ are at $z=\infty$. Computing, we find

$$
\begin{aligned}
T & =\frac{i}{\pi} \sum_{j} \partial \bar{\partial} h_{\lambda}\left(\tilde{c}_{j}(\lambda)\right) \\
& =(d-1) \frac{i}{\pi} \partial \bar{\partial} h_{\lambda}(0,1) \\
& =(d-1) \frac{i}{\pi} \partial \bar{\partial} G_{\lambda}(0) \\
& =\frac{d-1}{d} \frac{i}{\pi} \partial \bar{\partial} G_{\lambda}(\lambda) .
\end{aligned}
$$

Example 6.2. Let $f$ be a polynomial of degree $d$ and $G_{f}$ the Green's function for the complement of the filled Julia set. The Lyapunov exponent of $f$ (for the Brolin-Lyubich measure) satisfies ([Prz],[Mn])

$$
L(f)=\log d+\sum_{\left\{c \in \mathbf{C}: f^{\prime}(c)=0\right\}} G_{f}(c) .
$$


If $\left\{f_{\lambda}\right\}$ is any holomorphic family of polynomials, the Lyapunov exponent as a function of the parameter is a potential function for the bifurcation current; that is,

$$
T=\frac{i}{\pi} \partial \bar{\partial} L
$$

In the sequel, we examine further the connection between the bifurcation current and the Lyapunov exponent.

\section{References}

[BE] L. Bers and L. Ehrenpreis, Holomorphic convexity of Teichmüller spaces, Bull. Amer. Math. Soc 70 (1964), 761-764.

[B] H. Brolin, Invariant sets under iteration of rational functions. Ark. Mat. 6 (1965), 103-144.

[CG] L. Carleson and T. W. Gamelin, Complex dynamics, Springer-Verlag, New York, 1993.

[C] U. Cegrell, Plurisubharmonic functions, Proc. London Math. Soc. 36 (1978), 310-336.

[FS] J. E. Fornaess and N. Sibony, Complex dynamics in higher dimension. II. Modern methods in complex analysis (Princeton, NJ, 1992), Princeton Univ. Press, Princeton, NJ, 1995, pp. 135-182.

[GH] P. Griffiths and J. Harris, Principles of algebraic geometry, John Wiley \& Sons Inc., New York, 1994.

[H] L. Hörmander, An introduction to complex analysis in several variables, North-Holland Publishing Co., Amsterdam, 1990.

[HP] J. Hubbard and P. Papadopol, Superattractive fixed points in $\mathbf{C}^{n}$, Indiana Univ. Math. J. 43 (1994), 321-365.

[Le] P. Lelong, Fonctions plurisousharmoniques et formes différentielles positives, Gordon \& Breach, Paris, 1968.

[Ly] M. Lyubich, Entropy properties of rational endomorphisms of the Riemann sphere, Ergodic Theory Dynamical Systems 3 (1983), 351-385.

[Mn] R. Mañé, The Hausdorff dimension of invariant probabilities of rational maps, Dynamical systems, Valparaiso 1986, Lecture Notes in Math. 1331, Springer, Berlin, 1988, pp. 86-117.

[MSS] R. Mañé, P. Sad, and D. Sullivan, On the dynamics of rational maps, Ann. Sci. cole Norm. Sup. 16 (1983), 193-217.

[M1] C. McMullen, Complex dynamics and renormalization, Annals of Mathematics Studies 135, Princeton University Press, Princeton, NJ, 1994.

[M2] - Rational maps and Teichmüller space, Lecture Notes in Mathematics 1574, Springer-Verlag, 1994, pp. 430-433.

[Prz] F. Przytycki, Hausdorff dimension of harmonic measure on the boundary of an attractive basin for a holomorphic map, Invent. Math. 80 (1985), 161-179.

[R] R. M. Range, Holomorphic functions and integral representations in several complex variables, Graduate Texts in Mathematics 108, Springer-Verlag, New York, 1986.

[S] Y.-T. Siu, Pseudoconvexity and the Problem of Levi, Bull. Amer. Math. Soc. 84 (1978), 481-512.

[U] T. Ueda, Fatou sets in complex dynamics on projective spaces, J. Math. Soc. Japan 46 (1994), 545-555.

Department of Mathematics, Harvard University, One Oxford Street Cambridge, MA 02138.

E-mail address: demarco@math.harvard.edu 